\title{
Monopole Dominance of Confinement in SU(3) Lattice QCD
}

\author{
Hideo Suganuma*, \\ Department of Physics \& Division of Physics and Astronomy, Graduate School of Science, \\ Kyoto University, Kitashirakawaoiwake, Sakyo, Kyoto 606-8502, Japan \\ E-mail: suganumadscphys.kyoto-u.ac.jp
}

\section{Naoyuki Sakumichi}

Ochanomizu University, 2-1-1 Otsuka, Bunkyo, Tokyo 112-8610, Japan

To check the dual superconductor picture for the quark-confinement mechanism, we evaluate monopole dominance as well as Abelian dominance of quark confinement for both quarkantiquark (QQ̄) and three-quark (3Q) systems in SU(3) quenched lattice QCD in the maximally Abelian (MA) gauge. First, we examine Abelian dominance for the static QQ $\bar{Q}$ system in lattice QCD with various spacing $a$ at $\beta=5.8-6.4$ and various size $L^{3} \times L_{t}$. For large physicalvolume lattices with $L a \geq 2 \mathrm{fm}$, we find perfect Abelian dominance of the string tension for the QQ systems: $\sigma_{\text {Abel }} \simeq \sigma$. Second, we accurately measure the static 3Q potential for more than 300 different patterns of 3Q systems with 1000-2000 gauge configurations using two large physicalvolume lattices: $\left(\beta, L^{3} \times L_{t}\right)=\left(5.8,16^{3} \times 32\right)$ and $\left(6.0,20^{3} \times 32\right)$. For all the distances, the static $3 \mathrm{Q}$ potential is found to be well described by the Y-Ansatz, i.e., two-body Coulomb term plus three-body Y-type linear term $\sigma L_{\min }$, where $L_{\min }$ is the minimum flux-tube length connecting the three quarks. We find perfect Abelian dominance of the string tension also for the $3 \mathrm{Q}$ systems: $\sigma_{3 \mathrm{Q}}^{\mathrm{Abel}} \simeq \sigma_{3 \mathrm{Q}} \simeq \sigma$. Finally, we accurately investigate monopole dominance in SU(3) lattice QCD at $\beta=5.8$ on $16^{3} \times 32$ with 2,000 gauge configurations. Abelian-projected QCD in the MA gauge has not only the color-electric current $j^{\mu}$ but also the color-magnetic monopole current $k^{\mu}$, which topologically appears. By the Hodge decomposition, the Abelian-projected QCD system can be divided into the monopole part $\left(k_{\mu} \neq 0, j_{\mu}=0\right)$ and the photon part $\left(j_{\mu} \neq 0, k_{\mu}=0\right)$. We find monopole dominance of the string tension for QQ $\bar{Q}$ and $3 \mathrm{Q}$ systems: $\sigma_{\mathrm{Mo}} \simeq 0.92 \sigma$. While the photon part has almost no confining force, the monopole part almost keeps the confining force.

The XIIIth Quark Confinement and the Hadron Spectrum,

31 July - 6 August 2018

Maynooth University, Maynooth, Ireland

${ }^{*}$ Speaker. 


\section{Introduction: Dual Superconductor Picture and Maximally Abelian Gauge}

Quantum chromodynamics (QCD) is the fundamental theory of the strong interaction, but the QCD system is highly complicated and is still unsolved analytically because of its strong coupling in the low-energy region. In particular, quark confinement is an outstanding strange phenomenon exhibited in nonperturbative QCD, because the fundamental degrees of freedom, quarks and gluons, cannot be observed, and there is almost no similar phenomenon in other region of physics. In fact, to clarify the confinement mechanism is one of the most difficult important unsolved problems remaining in modern physics.

For the quark-confinement mechanism, Nambu, 't Hooft and Mandelstam proposed a dualsuperconductor picture in 1970's [1]. In this picture, the QCD vacuum is regarded as a colormagnetic monopole condensed system, and the dual Meissner effect forces the color-electric flux between (anti)quarks to be squeezed into one dimension, which leads to the flux-tube picture of hadrons [2, 3]. However, there are two large gaps between QCD and the dual-superconductor picture [4].

1. The dual-superconductor picture is based on the Abelian gauge theory subject to the Maxwelltype equations, but QCD is a non-Abelian gauge theory.

2. The dual-superconductor picture needs color-magnetic monopole condensation as the key concept, but QCD does not have such a monopole as the elementary degrees of freedom.

As a possible connection from QCD to the dual superconductor picture, 't Hooft proposed "Abelian projection" [2, 3] as an infrared Abelianization scheme of QCD. In the Abelian projection, magnetic monopoles topologically appear, and 't Hooft conjectured that long-distance physics like confinement is realized only by Abelian degrees of freedom in QCD [2], which is called "(infrared) Abelian dominance".

Actually, in the maximally Abelian (MA) gauge [5], off-diagonal gluons acquire a large effective mass of about $1 \mathrm{GeV}$ [6], which makes infrared QCD Abelian-like [7], and lattice QCD shows appearance of a large clustering of the monopole current covering the four-dimensional space-time [5, 8]. In fact, infrared QCD in the MA gauge seems to behave as an Abelian dual-superconductor.

In SU(3) lattice QCD, MA gauge fixing [9 10] is performed by maximizing

$$
R_{\mathrm{MA}}\left[U_{\mu}(s)\right] \equiv \sum_{s} \sum_{\mu=1}^{4} \operatorname{tr}\left(U_{\mu}^{\dagger}(s) \vec{H} U_{\mu}(s) \vec{H}\right)=\frac{1}{2} \sum_{s} \sum_{\mu=1}^{4}\left(\sum_{i=1}^{3}\left|U_{\mu}(s)_{i i}\right|^{2}-1\right),
$$

under $\mathrm{SU}(3)$ gauge transformation. Here, $U_{\mu}(s)$ is the link-variable $U_{\mu}(s)=e^{i a g A_{\mu}(s)} \in \mathrm{SU}(3)_{c}$ with lattice spacing $a$, gauge coupling $g$ and gluon fields $A_{\mu}$.

The Abelian link-variable $u_{\mu}(s)=e^{i \theta_{\mu}(s)}=e^{i \theta_{\mu}^{3}(s) T_{3}+i \theta_{\mu}^{8}(s) T_{8}} \in \mathrm{U}(1)^{2}$ is extracted from the link-variable $U_{\mu}^{\mathrm{MA}}(s) \in \mathrm{SU}(3)_{c}$ in the MA gauge [10], by maximizing the overlap of $R_{\text {Abel }} \equiv$ $\frac{1}{3} \operatorname{Retr}\left(U_{\mu}^{\mathrm{MA}}(s) u_{\mu}^{\dagger}(s)\right) \in\left[-\frac{1}{2}, 1\right]$. Maximally Abelian projection is defined by the replacement of $\left\{U_{\mu}^{\mathrm{MA}}(s)\right\} \rightarrow\left\{u_{\mu}(s)\right\}$, which corresponds to the elimination of off-diagonal gluon components.

In this paper, to check the dual superconductor picture, we accurately investigate Abelian dominance [9 10] and monopole dominance of the quark confining force for both quark-antiquark $(\mathrm{QQ})$ and three-quark (3Q) systems in SU(3) quenched lattice QCD in the MA gauge. For the error estimate, we use the jackknife method. 


\section{Perfect Abelian dominance of quark confinement in quark-antiquark systems}

First, we study the static QQ $\bar{Q}$ potential $V(r)$ in lattice QCD with $\left(\beta, L^{3} \times L_{t}\right)=\left(6.4,32^{4}\right),\left(6.0,32^{4}\right)$ and $\left(5.8,16^{3} \times 32\right)$ 9 10]. The QQ potential $V(r)$ is obtained with the Wilson loop $W_{r \times t}\left[U_{\mu}\right]$, and its MA projection (Abelian part) $V_{\text {Abel }}(r)$ is similarly defined by the Abelian Wilson loop $W_{r \times t}\left[u_{\mu}\right]$,

$$
V(r)=-\lim _{t \rightarrow \infty} \frac{1}{t} \ln \left\langle W_{r \times t}\left[U_{\mu}\right]\right\rangle, \quad V_{\text {Abel }}(r)=-\lim _{t \rightarrow \infty} \frac{1}{t} \ln \left\langle W_{r \times t}\left[u_{\mu}\right]\right\rangle .
$$

We show in Fig.1(a) the QQ̄ potential $V(r)$ and its Abelian part $V_{\text {Abel }}(r)$. They are found to be well reproduced by the Coulomb-plus-linear Ansatz, respectively:

$$
V(r)=-\frac{A}{r}+\sigma r+C, \quad V_{\text {Abel }}(r)=-\frac{A_{\text {Abel }}}{r}+\sigma_{\text {Abel }} r+C_{\text {Abel }} .
$$

Figure 1(b) shows the difference $V(r)-V_{\text {Abel }}(r)$ plotted with $r$ at each lattice. As a remarkable fact from Fig.1, we find perfect Abelian dominance of the string tension, $\sigma_{\text {Abel }} \simeq \sigma$, for the $\mathrm{QQ}$ system.
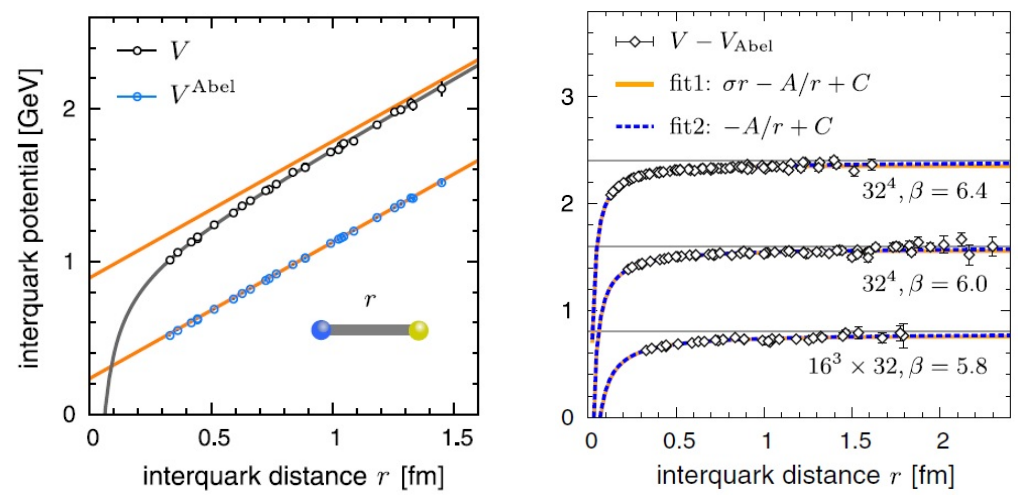

Figure 1: (a) The lattice QCD result of the QQ potential $V(r)$ (black) and its Abelian part $V_{\text {Abel }}(r)$ (blue) for $\left(\beta, L^{3} L_{t}\right)=\left(5.8,16^{3} 32\right)$ [10]. (b) $V(r)-V_{\text {Abel }}(r)$ for $\left(\beta, L^{3} L_{t}\right)=\left(6.4,32^{4}\right),\left(6.0,32^{4}\right)$ and $\left(5.8,16^{3} 32\right)$ [9. At each lattice, all the data can be well fit with the pure Coulomb form with $\sigma=0$, which means $\sigma_{\text {Abel }} \simeq \sigma$.

We also examine the physical lattice-volume dependence of $\sigma_{\mathrm{Abel}} / \sigma$ in Fig.2, and find perfect Abelian dominance $\left(\sigma_{\text {Abel }} \simeq \sigma\right.$ ), when the spatial size $L a$ is larger than about $2 \mathrm{fm}$.

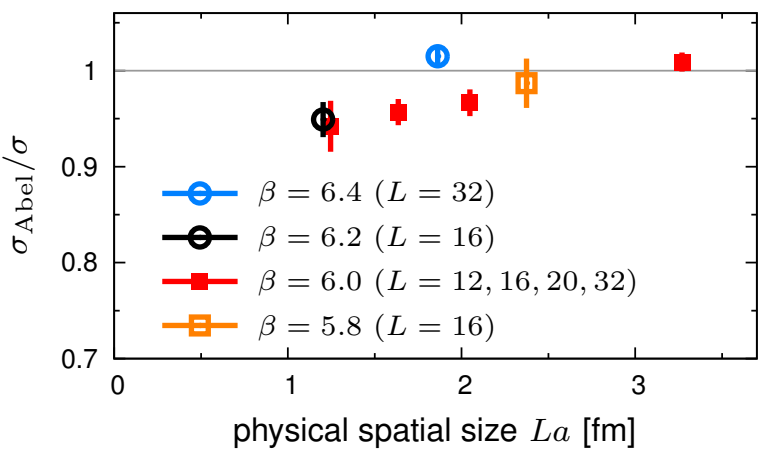

Figure 2: Physical spatial-size dependence of the ratio $\sigma_{\text {Abel }} / \sigma[10]$. Perfect Abelian dominance $\left(\sigma_{\text {Abel }} \simeq \sigma\right)$ is found for larger lattices with $L a \geq 2 \mathrm{fm}$. 


\section{Quark Confinement in Baryons}

Second, we accurately measure the static three-quark (3Q) potential $V_{3 \mathrm{Q}}$ [10] for more than 300 different patterns of 3Q systems with 1000-2000 gauge configurations in SU(3) lattice QCD on large physical-volume lattices with $L a>2 \mathrm{fm}:\left(\beta, L^{3} \times L_{t}\right)=\left(5.8,16^{3} \times 32\right)$ and $\left(6.0,20^{3} \times 32\right)$.

\subsection{Accurate Measurement of Three-Quark Potential}

Like the QQ potential, the $3 \mathrm{Q}$ potential $V_{3 \mathrm{Q}}$ is obtained from the "3Q Wilson loop" $W_{3 \mathrm{Q}}$ (an extension of the Wilson loop for gauge-invariant static 3Q systems) defined in Ref.[11]:

$$
V_{3 \mathrm{Q}}=-\lim _{t \rightarrow \infty} \frac{1}{t} \ln \left\langle W_{3 \mathrm{Q}}\left[U_{\mu}\right]\right\rangle .
$$

We consider 101 and 211 different patterns of 3Q systems with 2000 and 1000 gauge configurations at $\beta=5.8$ and 6.0, respectively. For the accurate calculation of the 3Q potential with finite $t$, we use the gauge-invariant smearing method [11], which enhances the ground-state component in the $3 \mathrm{Q}$ state in $\left\langle W_{3 \mathrm{Q}}\right\rangle$.

As the result, all the lattice QCD data of the $3 \mathrm{Q}$ potential $V_{3 \mathrm{Q}}$ is found to be fairly well reproduced by the Y-Ansatz [10,11], i.e., one-gluon-exchange Coulomb plus Y-type linear potential,

$$
V_{3 \mathrm{Q}}\left(\mathbf{r}_{1}, \mathbf{r}_{2}, \mathbf{r}_{3}\right)=-\sum_{i<j} \frac{A_{3 \mathrm{Q}}}{\left|\mathbf{r}_{i}-\mathbf{r}_{j}\right|}+\sigma_{3 \mathrm{Q}} L_{\min }+C_{3 \mathrm{Q}}=-\frac{A_{3 \mathrm{Q}}}{R}+\sigma_{3 \mathrm{Q}} L_{\min }+C_{3 \mathrm{Q}}
$$

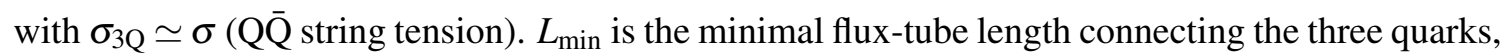
located at $\mathbf{r}_{1}, \mathbf{r}_{2}$ and $\mathbf{r}_{3}$. We here introduce a convenient variable $1 / R \equiv \sum_{i<j} 1 /\left|\mathbf{r}_{i}-\mathbf{r}_{j}\right|$ [12]. The Y-Ansatz (3.2) indicates the Y-shaped flux-tube formation in baryons, which is actually observed in lattice QCD calculations on the action density in the presence of three static quarks [13].

\subsection{Perfect Abelian dominance of quark confinement in baryons}

Next, we examine Abelian dominance of quark confinement in the 3Q system. Like the QQ case, the MA-projected 3Q potential $V_{3 \mathrm{Q}}^{\text {Abel }}$ (Abelian part) is defined by the Abelian 3Q Wilson loop $W_{3 \mathrm{Q}}\left[u_{\mu}\right]$ in the MA gauge,

$$
V_{3 \mathrm{Q}}^{\mathrm{Abel}}=-\lim _{t \rightarrow \infty} \frac{1}{t} \ln \left\langle W_{3 \mathrm{Q}}\left[u_{\mu}\right]\right\rangle .
$$

Figure 3(a) shows the $3 \mathrm{Q}$ potential $V_{3 \mathrm{Q}}$ and its Abelian part $V_{3 \mathrm{Q}}^{\text {Abel }}$ plotted against the total fluxtube length $L_{\min }$ in $\mathrm{SU}(3)$ lattice QCD at $\beta=5.8$ on $16^{3} \times 32$ with 2,000 gauge configurations [10].

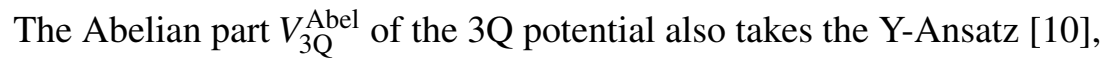

$$
V_{3 \mathrm{Q}}^{\mathrm{Abel}}=-\sum_{i<j} \frac{A_{3 \mathrm{Q}}^{\mathrm{Abel}}}{\left|\mathbf{r}_{i}-\mathbf{r}_{j}\right|}+\sigma_{3 \mathrm{Q}}^{\mathrm{Abel}} L_{\mathrm{min}}+C_{3 \mathrm{Q}}^{\mathrm{Abel}}=-\frac{A_{3 \mathrm{Q}}^{\mathrm{Abel}}}{R}+\sigma_{3 \mathrm{Q}}^{\mathrm{Abel}} L_{\mathrm{min}}+C_{3 \mathrm{Q}}^{\mathrm{Abel}},
$$

with $1 / R \equiv \sum_{i<j} 1 /\left|\mathbf{r}_{i}-\mathbf{r}_{j}\right|$. At long distances, $V_{3 \mathrm{Q}}$ and $V_{3 \mathrm{Q}}^{\text {Abel }}$ are almost single-valued functions of $L_{\min }$, although their multi-valued feature due to the $R$-dependence is more visible at short distances on finer lattices at $\beta=6.0$. From Fig.3(a), we find perfect Abelian dominance also for the 3Q confinement force, i.e., $\sigma_{3 \mathrm{Q}}^{\text {Abel }} \simeq \sigma_{3 \mathrm{Q}} \simeq \sigma$. 

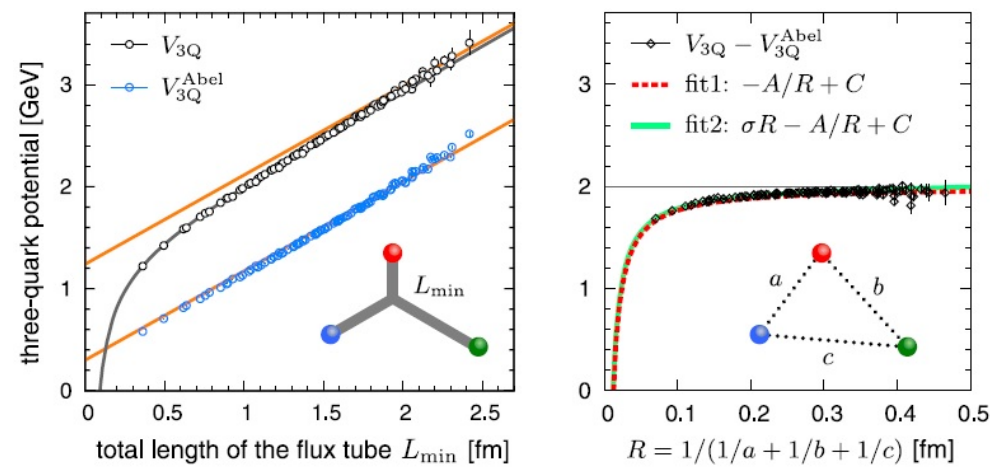

Figure 3: (a) The $3 \mathrm{Q}$ potential $V_{3 \mathrm{Q}}$ (black) and its Abelian part $V_{3 \mathrm{Q}}^{\text {Abel }}$ (blue) plotted against $L_{\min }$ in $\mathrm{SU}(3)$ lattice QCD at $\beta=5.8$ on $16^{3} \times 32$. We add the best-fit Y-Ansatz curve of the equilateral 3Q case for $V_{3 \mathrm{Q}}$ and $V_{3 \mathrm{Q}}^{\text {Abel }}$, respectively. (b) $\Delta V_{3 \mathrm{Q}} \equiv V_{3 \mathrm{Q}}-V_{3 \mathrm{Q}}^{\text {Abel }}$ plotted against $R . \Delta V_{3 \mathrm{Q}}$ can be fit with the pure Coulomb Ansatz (3.4) with no string tension, which indicates $\sigma_{3 \mathrm{Q}} \simeq \sigma_{3 \mathrm{Q}}^{\mathrm{Abel}}$. These figures are taken from Ref.[10].

To demonstrate $\sigma_{3 \mathrm{Q}}^{\text {Abel }} \simeq \sigma_{3 \mathrm{Q}}$, we show in Fig.3(b) the difference $\Delta V_{3 \mathrm{Q}} \equiv V_{3 \mathrm{Q}}-V_{3 \mathrm{Q}}^{\text {Abel }}$ plotted against $R \llbracket 10$, because, if $\sigma_{3 \mathrm{Q}}^{\text {Abel }}=\sigma_{3 \mathrm{Q}}, \Delta V_{3 \mathrm{Q}}$ is well reproduced by the pure Coulomb Ansatz,

$$
\Delta V_{3 \mathrm{Q}} \equiv V_{3 \mathrm{Q}}-V_{3 \mathrm{Q}}^{\mathrm{Abel}}=-\frac{\Delta A_{3 \mathrm{Q}}}{R}+\Delta C_{3 \mathrm{Q}},
$$

with $\Delta A_{3 \mathrm{Q}} \equiv A_{3 \mathrm{Q}}-A_{3 \mathrm{Q}}^{\mathrm{Abel}}, \Delta C_{3 \mathrm{Q}} \equiv C_{3 \mathrm{Q}}-C_{3 \mathrm{Q}}^{\mathrm{Abel}}$ and $1 / R \equiv \sum_{i<j} 1 /\left|\mathbf{r}_{i}-\mathbf{r}_{j}\right|$. In Fig.3(b), $\Delta V_{3 \mathrm{Q}}$ obeys a pure Coulomb form with no string tension, which is a clear evidence of perfect Abelian dominance of quark confinement in baryons: $\sigma_{3 \mathrm{Q}}^{\text {Abel }} \simeq \sigma_{3 \mathrm{Q}}$ [10].

To summarize, from the analysis of the accurate lattice QCD data of $V(r), V^{\mathrm{Abel}}(r), V_{3 \mathrm{Q}}$ and $V_{3 \mathrm{Q}}^{\mathrm{Abel}}$ [9], we find perfect Abelian dominance of the string tension in QQ $\overline{\mathrm{Q}}$ and 3Q potentials: $\sigma \simeq \sigma^{\text {Abel }} \simeq \sigma_{3 \mathrm{Q}} \simeq \sigma_{3 \mathrm{Q}}^{\text {Abel }}$.

\section{Monopole Dominance of Quark Confinement in Mesons and Baryons}

Finally, we accurately investigate monopole dominance of quark confinement for both QQ $\bar{Q}$ and 3Q systems in $\mathrm{SU}(3)$ lattice QCD at $\beta=5.8$ on $16^{3} \times 32$ with 2,000 gauge configurations.

\subsection{Hodge Decomposition and Monopole Projection}

In the MA gauge, it is likely that only Abelian gluon component is essential for the longdistance QCD physics, and infrared QCD can be approximated by Abelian-projected QCD, as is indicated by perfect Abelian dominance of quark confinement.

Abelian-projected QCD in the MA gauge has not only the color-electric current $j^{\mu}$ but also the color-magnetic monopole current $k^{\mu}$, which topologically appears. In the dual superconductor scenario, the monopole current $k^{\mu}$ is considered to play an essential role to quark confinement. By the Hodge decomposition, the Abelian-projected QCD system can be divided into the monopole part $\left(k_{\mu} \neq 0, j_{\mu}=0\right)$ and the photon part $\left(j_{\mu} \neq 0, k_{\mu}=0\right)$, as schematically illustrated in Fig.4. Then, the importance of the monopole current $k^{\mu}$ can be checked, using the Hodge decomposition.

In the lattice formalism, DeGrand and Toussaint performed the Hodge decomposition [14]. In lattice QCD, the Abelian gluon $\theta_{\mu}(s)=a g A_{\mu}(s)$ is the exponent in Abelian link-variable,

$$
u_{\mu}(s)=e^{i \theta_{\mu}(s)}=e^{i \theta_{\mu}^{3}(s) T^{3}+i \theta_{\mu}^{8}(s) T^{8}}=\operatorname{diag}\left(e^{i \theta_{\mu}^{(1)}(s)}, e^{i \theta_{\mu}^{(2)}(s)}, e^{i \theta_{\mu}^{(3)}(s)}\right) \in \mathrm{U}(1)^{2},
$$




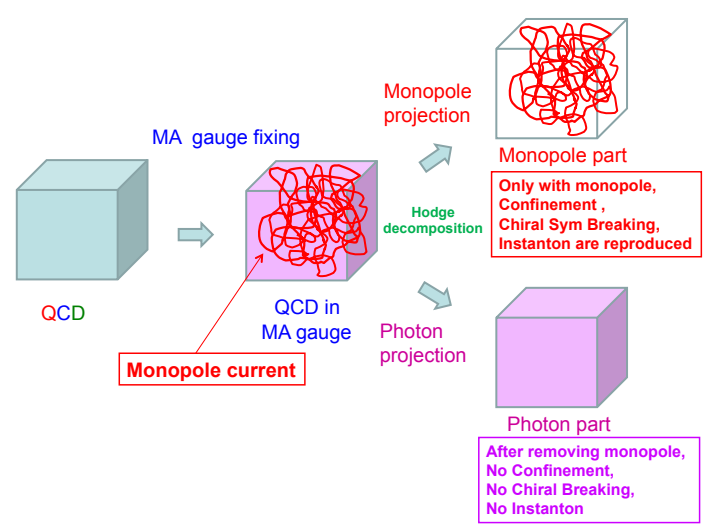

Figure 4: A dual superconductor scenario from QCD in the MA gauge. In the MA gauge, infrared QCD becomes Abelian-like because of large off-diagonal gluon mass of about $1 \mathrm{GeV}$ [6], and monopole currents topologically appear [5]. By the Hodge decomposition, the QCD system in the MA gauge can be divided into the monopole part $\left(k_{\mu} \neq 0, j_{\mu}=0\right)$ and the photon part $\left(j_{\mu} \neq 0, k_{\mu}=0\right)$.

with $\theta_{\mu}^{(i)}(s) \in[-\pi, \pi)(i=1,2,3)$, which is consistent with the continuum gluon $A_{\mu}$ as $a \rightarrow 0$. The Abelian field strength $\theta_{\mu v}(s)=a^{2} g F_{\mu \nu}(s)$ is the exponent in the Abelian plaquette variable,

$$
u_{\mu v}(s)=e^{i(\partial \wedge \theta)_{\mu v}(s)}=e^{i \theta_{\mu v}(s)}=e^{i \theta_{\mu v}^{3}(s) T^{3}+i \theta_{\mu \nu}^{8}(s) T^{8}}=\operatorname{diag}\left(e^{i \theta_{\mu \nu}^{(1)}(s)}, e^{i \theta_{\mu \nu}^{(2)}(s)}, e^{i \theta_{\mu \nu}^{(3)}(s)}\right) \in \mathrm{U}(1)^{2} .
$$

Here, $\theta_{\mu \nu}^{(i)}(s)$ is the principal value $\theta_{\mu \nu}^{(i)}(s) \in[-\pi, \pi)(i=1,2,3)$, which is $\mathrm{U}(1)^{2}$-gauge invariant and consistent with the continuum Abelian field strength $F_{\mu \nu}$ as $a \rightarrow 0$ [4]. Then, $\theta_{\mu v}$ is written as

$$
\theta_{\mu v}(s)=(\partial \wedge \theta)_{\mu v}(s)+2 \pi n_{\mu v}(s), \quad n_{\mu v}^{(i)}(s) \in \mathbf{Z} \quad(i=1,2,3),
$$

where $n_{\mu v}(s)$ is $\mathrm{U}(1)^{2}$ gauge-variant and corresponds to the singular Dirac string as $a \rightarrow 0$ [4]. The electric current $j^{\mu}$ and the monopole current $k^{\mu}$ are derived from the Abelian field strength $\theta_{\mu \nu}$,

$$
j_{v} \equiv \partial_{\mu} \theta_{\mu v}, \quad k_{v} \equiv \partial_{\mu} \tilde{\theta}_{\mu v}=2 \pi \partial_{\mu} \tilde{n}_{\mu v}, \quad k_{v}^{(i)}=2 \pi \partial_{\mu} \tilde{n}_{\mu \nu}^{(i)} \in 2 \pi \mathbf{Z},
$$

with the dual tensor $\tilde{\theta}_{\mu v} \equiv \frac{1}{2} \varepsilon_{\mu v \alpha \beta} \theta_{\alpha \beta}$. The monopole part $\theta_{\mu}^{\mathrm{Mo}}$ and the photon part $\theta_{\mu}^{\mathrm{Ph}}$ satisfy

$$
\begin{aligned}
& \theta_{\mu \nu}^{\mathrm{Mo}} \equiv\left(\partial \wedge \theta^{\mathrm{Mo}}\right)_{\mu \nu}(\bmod 2 \pi), \quad \partial_{\mu} \theta_{\mu \nu}^{\mathrm{Mo}}=0, \quad \partial_{\mu} \tilde{\theta}_{\mu \nu}^{\mathrm{Mo}}=k_{v}, \\
& \theta_{\mu \nu}^{\mathrm{Ph}} \equiv\left(\partial \wedge \theta^{\mathrm{Ph}}\right)_{\mu \nu} \quad(\bmod 2 \pi), \quad \partial_{\mu} \theta_{\mu \nu}^{\mathrm{Ph}}=j_{\nu}, \quad \partial_{\mu} \tilde{\theta}_{\mu \nu}^{\mathrm{Ph}}=0 .
\end{aligned}
$$

From $\partial_{\mu} \tilde{\theta}_{\mu \nu}^{\mathrm{Ph}}=0$, one finds $\theta_{\mu \nu}^{\mathrm{Ph}}=\left(\partial \wedge \theta^{\mathrm{Ph}}\right)_{\mu \nu}$ and $\partial_{\mu}\left(\partial \wedge \theta^{\mathrm{Ph}}\right)_{\mu \nu}=\partial^{2} \theta_{v}^{\mathrm{Ph}}-\partial_{v}\left(\partial_{\mu} \theta_{\mu}^{\mathrm{Ph}}\right)=j_{v}$. Taking the Landau gauge $\partial_{\mu} \theta_{\mu}^{\mathrm{Ph}}=0$, the photon part $\theta_{v}^{\mathrm{Ph}}$ is derived from the electric current $j_{v}$,

$$
\partial^{2} \theta_{v}^{\mathrm{Ph}}=j_{v}, \quad \theta_{v}^{\mathrm{Ph}}=\frac{1}{\partial^{2}} j_{v}, \quad \text { i.e. }, \quad \theta_{v}^{\mathrm{Ph}}(s)=\sum_{s^{\prime}}\left\langle s\left|\frac{1}{\partial^{2}}\right| s^{\prime}\right\rangle j_{v}\left(s^{\prime}\right),
$$

using the inverse d'Alembertian on the lattice [4]. The monopole part $\theta_{\mu}^{\mathrm{Mo}}(s)$ is obtained as $\theta_{\mu}^{\mathrm{Mo}}(s)=\theta_{\mu}(s)-\theta_{\mu}^{\mathrm{Ph}}(s)$. The monopole part $\theta_{\mu}^{\mathrm{Mo}}(s)$ and the photon part $\theta_{\mu}^{\mathrm{Ph}}(s)$ satisfy Eqs. (4.5) and (4.6) near the continuum with a small $a$ [4].

Using the monopole/photon link-variables,

$$
u_{\mu}^{\mathrm{Mo}}(s) \equiv e^{i \theta_{\mu}^{\mathrm{Mo}}(s)} \in \mathrm{U}(1)^{2}, \quad u_{\mu}^{\mathrm{Ph}}(s) \equiv e^{i \theta_{\mu}^{\mathrm{Ph}}(s)} \in \mathrm{U}(1)^{2},
$$

monopole projection and photon projection are defined as follows: 
- Monopole projection (the monopole part) is defined by the replacement of $\left\{u_{\mu}(s)\right\} \rightarrow\left\{u_{\mu}^{\mathrm{Mo}}(s)\right\}$, which keeps the monopole current $k^{\mu}$ and eliminates the electric current $j^{\mu}$.

- Photon projection (the photon part) is defined by the replacement of $\left\{u_{\mu}(s)\right\} \rightarrow\left\{u_{\mu}^{\mathrm{Ph}}(s)\right\}$, which keeps the electric current $j^{\mu}$ and eliminates the monopole current $k^{\mu}$.

The dominant role of the monopole part is called "monopole dominance", and monopole dominance has been investigated for quark confinement in lattice QCD [8].

\subsection{Monopole Dominance of Confinement for Quark-Antiquark and 3Q Systems}

The monopole part $V_{\mathrm{Mo}}(r)$ and the photon part $V_{\mathrm{Ph}}(r)$ of the QQ potential are defined by the monopole/photon-projected Wilson loop, $W_{r \times t}\left[u_{\mu}^{\mathrm{Mo}}\right]$ and $W_{r \times t}\left[u_{\mu}^{\mathrm{Ph}}\right]$,

$$
V_{\mathrm{Mo}}(r)=-\lim _{t \rightarrow \infty} \frac{1}{t} \ln \left\langle W_{r \times t}\left[u_{\mu}^{\mathrm{Mo}}\right]\right\rangle, \quad V_{\mathrm{Ph}}(r)=-\lim _{t \rightarrow \infty} \frac{1}{t} \ln \left\langle W_{r \times t}\left[u_{\mu}^{\mathrm{Ph}}\right]\right\rangle .
$$

Figure 5(a) shows the lattice QCD result for the static QQ potential $V(r)$ in $\mathrm{SU}(3) \mathrm{QCD}, V_{\text {Abel }}(r)$ in Abelian-projected QCD, $V_{\mathrm{Mo}}(r)$ in the monopole part, and $V_{\mathrm{Ph}}(r)$ in the photon part. While the photon part has almost no confining force, the monopole part almost keeps the confining force. Thus, monopole dominance is found for quark confinement in the QQ $\bar{Q}$ system.
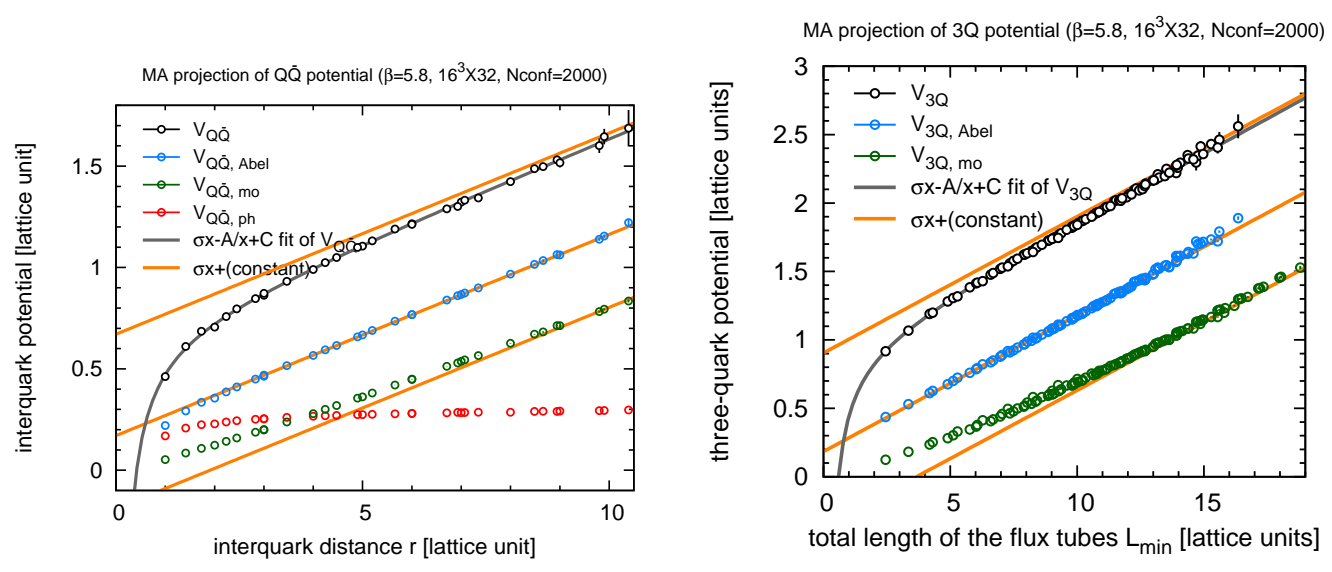

Figure 5: (a) The lattice QCD result for the static QQ potential $V(r)$ (black) in $\mathrm{SU}(3) \mathrm{QCD}, V_{\text {Abel }}(r)$ (blue) in Abelian-projected QCD, $V_{\mathrm{Mo}}(r)$ (green) in the monopole part, and $V_{\mathrm{Ph}}(r)$ (red) in the photon part. (b) The lattice QCD result for the $3 \mathrm{Q}$ potential $V_{3 \mathrm{Q}}$ (black) in SU(3) QCD, $V_{3 \mathrm{Q}}^{\text {Abel }}$ (blue) in Abelian-projected QCD, and $V_{3 \mathrm{Q}}^{\mathrm{Mo}}$ (green) in the monopole part, plotted against $L_{\min }$.

Similarly, the monopole part $V_{3 \mathrm{Q}}^{\mathrm{Mo}}(r)$ of the $3 \mathrm{Q}$ potential is defined by

$$
V_{3 \mathrm{Q}}^{\mathrm{Mo}}=-\lim _{t \rightarrow \infty} \frac{1}{t} \ln \left\langle W_{3 \mathrm{Q}}\left[u_{\mu}^{\mathrm{Mo}}\right]\right\rangle .
$$

Figure 5(b) shows the lattice QCD result for the 3Q potential $V_{3 \mathrm{Q}}$ in $\mathrm{SU}(3) \mathrm{QCD}, V_{3 \mathrm{Q}}^{\text {Abel }}$ in Abelianprojected QCD, and $V_{3 \mathrm{Q}}^{\mathrm{Mo}}$ in the monopole part, plotted against $L_{\mathrm{min}}$. Monopole dominance is found also for quark confinement in the $3 \mathrm{Q}$ system.

The string tension $\sigma_{\mathrm{Mo}}$ in the monopole part is estimated from the lattice QCD data in Fig.5, and monopole dominance is estimated as $\sigma_{\mathrm{Mo}} \simeq 0.92 \sigma$ for the string tension in $\mathrm{QQ}$ and $3 \mathrm{Q}$ systems. 


\section{Summary and concluding remarks}

We have investigated Abelian dominance and monopole dominance of quark confinement for $\mathrm{QQ}$ and 3Q systems in SU(3) quenched lattice QCD in the MA gauge. For large physical-volume lattices with $L a \geq 2 \mathrm{fm}$, we have found perfect Abelian dominance of the string tension for both $\mathrm{QQ}$ and 3Q systems: $\sigma \simeq \sigma^{\text {Abel }} \simeq \sigma_{3 \mathrm{Q}} \simeq \sigma_{3 \mathrm{Q}}^{\text {Abel }}$. We have found monopole dominance of the string tension for both $\mathrm{QQ}$ and $3 \mathrm{Q}$ systems, and have estimated $\sigma_{\mathrm{Mo}} \simeq 0.92 \sigma$.

\section{Acknowledgments}

H.S. is supported in part by the Grants-in-Aid for Scientific Research [15K05076] from Japan Society for the Promotion of Science. The lattice QCD calculations were done on NEC-SX8R at Osaka University.

\section{References}

[1] Y. Nambu, Phys. Rev. D10, 4262 (1974); G. 't Hooft, in High Energy Physics, (Editorice Compositori, Bologna, 1975); S. Mandelstam, Phys. Rept. 23, 245 (1976).

[2] G. 't Hooft, Nucl. Phys. B190, 455 (1981).

[3] Z. F. Ezawa and A. Iwazaki, Phys. Rev. D25, 2681 (1982).

[4] H. Ichie and H. Suganuma, Nucl. Phys. B548, 365 (1999); Nucl. Phys. B574, 70 (2000); H. Suganuma, H. Ichie, A. Tanaka and K. Amemiya, Prog. Theor. Phys. Suppl. 131, 559 (1998).

[5] A. S. Kronfeld, G. Schierholz and U.-J. Wiese, Nucl. Phys. B293 461 (1987); A. S. Kronfeld, M. L. Laursen, G. Schierholz and U.-J. Wiese, Phys. Lett. B198, 516 (1987).

[6] K. Amemiya and H. Suganuma, Phys. Rev. D60, 114509 (1999); S. Gongyo and H. Suganuma, Phys. Rev. D87, 074506 (2013); S. Gongyo, T. Iritani and H. Suganuma, Phys. Rev. D86, 094018 (2012).

[7] T. Suzuki and I. Yotsuyanagi, Phys. Rev. D42, 4257(R) (1990).

[8] J. D. Stack, S. D. Neiman and R. J. Wensley, Phys. Rev. D50, 3399 (1994).

[9] N. Sakumichi and H. Suganuma, Phys. Rev. D90, 111501(R) (2014).

[10] N. Sakumichi and H. Suganuma, Phys. Rev. D92, 034511 (2015).

[11] T. T. Takahashi, H. Matsufuru, Y. Nemoto and H. Suganuma, Phys. Rev. Lett. 86, 18 (2001); T. T. Takahashi, H. Suganuma, Y. Nemoto and H. Matsufuru, Phys. Rev. D65, 114509 (2002); T. T. Takahashi and H. Suganuma, Phys. Rev. Lett. 90, 182001 (2003); Phys. Rev. D70, 074506 (2004).

[12] N. Brambilla, J. Ghiglieri and A. Vairo, Phys. Rev. D81, 054031 (2010); N. Brambilla, F. Karbstein and A. Vairo, Phys. Rev.D87, 074014 (2013).

[13] H. Ichie, V. Bornyakov, T. Streuer and G. Schierholz, Nucl. Phys. A721, 899 (2003); V. G. Bornyakov et al. (DIK Coll.), Phys. Rev. D70, 054506 (2004); Phys. Rev D70, 074511 (2004).

[14] T. A. DeGrand and D. Toussaint, Phys. Rev. D22, 2478 (1980). 